\title{
Fifteen Years Experience of Managing Penetrating Extra-Peritoneal Rectal Injuries
}

\author{
Tanzeela Gala ${ }^{1}$, Noman Shahzad ${ }^{2 *}$, Ahmed Ibrahim Edhi ${ }^{3}$, Fareed Ahmed Shaikh ${ }^{4}$ and Hasnain Zafar \\ ${ }^{1}$ Associate Specialist, Department of Colorectal Surgery, Royal Free London NHS Foundation Trust, UK \\ ${ }^{2}$ Consultant General Surgeon, Aga Khan University Hospital Karachi, Pakistan \\ ${ }^{3}$ Medical Student, Aga Khan University Hospital Karachi, Pakistan \\ ${ }^{4}$ Consultant Vascular Surgeon, Aga Khan University Hospital Karachi, Pakistan
}

${ }^{5}$ Associate Professor Trauma Surgery, Aga Khan University Hospital Karachi, Pakistan

*Corresponding author: Noman Shahzad, Consultant General Surgeon, Aga Khan University Hospital Karachi

Submission: April 26, 2018; Published: May 03, 2018

\begin{abstract}
Fifteen Years Experience of Managing Penetrating Extra-peritoneal Rectal Injuries.

Background: Although civilian injuries are generally less severe, they nevertheless remain a challenging problem for the surgeons. In isolated rectal injury patients, though proximal diversion, pre-sacral drainage, distal rectal wash-out and wound debridement are the various surgical options employed in various combinations, the optimum strategy especially for civilian injuries remains unknown. We reviewed our experience of managing penetrating extra peritoneal rectal injuries.
\end{abstract}

Methods: We conducted a Retrospective review of Adult patients with penetrating extra-peritoneal rectal injuries. Follow-up information of at least one month was needed for early post-operative complications.

Results: A total number of fifteen patients met inclusion criteria. Median age of our patients was 46 years with range being 20-80 years. All our patients were males. Thirteen of our patients (86\%) suffered from gunshot injury while one was a blast victim and one had a stab injury to rectum. Nine patients $(60 \%)$ had pelvic fracture associated with rectal injury.

Diversion stoma was made in all of our patients. Overall post-operative morbidity was $40 \%$. Two patients developed necrotizing fasciitis and required repeated debridements followed by graft placement and one patient developed intra-abdominal abscess which was treated by radiological guided drain placement and antibiotics.

Conclusion: Drainage with fecal diversion is the most commonly employed management of extra-peritoneal rectal injuries. Delayed or inadequate drainage can lead to disastrous consequences including necrotizing fasciitis, intra-abdominal abscess

Keywords: Extra-peritoneal rectal injury; Penetrating trauma; Diversion colostomy; Necrotizing fasciitis

\section{Introduction}

Rectal injury, alone or combined with colonic injury, is reported in $1.1 \%$ patients with trauma. Penetrating trauma accounts for $80 \%$ of those rectal injuries [1]. Principles of management of civilian rectal injuries have been adopted from knowledge gained from warfare. During the U.S. Civil War, penetrating abdominal injuries were often managed expectantly with mortality rate being as high as $90 \%$ [2]. With experience gained during World War I there was an improvement in survival from penetrating rectal injuries, when surgeons first began performing fecal diversion with creation of stoma. However, the mortality still remained as high as 50\% [3]. The mortality rate decreased further to $30 \%$ during World War
II with the introduction of trans-perineal pre-sacral drainage [4]. With the addition of distal washout during the Vietnam War, there was further improvement in care resulting in mortality associated with penetrating rectal injuries as low as 6\% [5].

Experience gained from management of warfare rectal injuries lead to the widely accepted doctrine that all penetrating rectal injuries require diversion, distal washout, and pre sacral drainage. Over decades, improved surgical techniques including damage control laparotomy and better understanding of management of the trauma patients have resulted in substantial reductions in morbidity and mortality [6]. Increasing use of Computerized 
Tomographic (CT) scan in trauma patients has also played role in making surgical plans well ahead in time [7].

Although civilian injuries are generally less severe, they nevertheless remain a challenging problem for the surgeons. In isolated rectal injury patients, though proximal diversion, presacral drainage, distal rectal wash-out and wound debridement are the various surgical options employed in various combinations, the optimum strategy especially for civilian injuries remains unknown. We reviewed our experience of managing penetrating extra peritoneal rectal injuries.

\section{Methods}

\section{Study design}

We conducted a Retrospective review of data.

\section{Study settings}

Study was conducted at Aga Khan University Hospital (AKUH) Karachi, Pakistan. AKUH is a tertiary care hospital with dedicated trauma surgeon and associated team. The hospital receives trauma patients from a wide area including metropolitan city of Karachi specifically and rest of country in general.

\section{Study duration}

Study was conducted from October 2003 till November 2017.

\section{Selection criteria}

Adult patients with penetrating extra-peritoneal rectal injuries were included in the study.

\section{Exclusion Criteria}

- $\quad$ Missing records

- $\quad$ Received post-operatively from other hospitals

\section{Data collection procedure}

Patients with penetrating extra-peritoneal rectal injuries were identified using ICD codes and data was retrieved from medical records. Data was collected regarding demographics, injuries, intra-operative details, post-operative stay and complications. Data was collected by two collectors to minimize errors. Followup information of at least one month was needed for early postoperative complications.

\section{Ethical considerations}

Approval was sought from institutional ethical review committee (2475-Sur-ERC-13)

\section{Statistical analysis}

Data entry and analysis was done in SPSS version 19. For continuous non-uniform data median with interquartile ranges are calculated. Mann-Whitney-U test and Kruskall-Wallis tests are used to compare data between two and greater than two groups. For dichotomous variables proportions and percentages are calculated while their analysis is done using Chi-square and Fischer's exact test. Uni-variate and multivariable logistic Regression Analysis is done to find out predictors of post-operative morbidity in our patients. Significance was defined as p-value $<0.05$.

\section{Results}

A total number of fifteen patients met inclusion criteria. Median age of our patients was 46 years with range being 20-80 years. Seven $(46 \%)$ of ours patients were older than 40 years of age. All our patients were males. Thirteen of our patients (86\%) suffered from gunshot injury while one was a blast victim and one had a stab injury to rectum. Median time for our patients to present to the hospital from time of injury was 187 minutes.

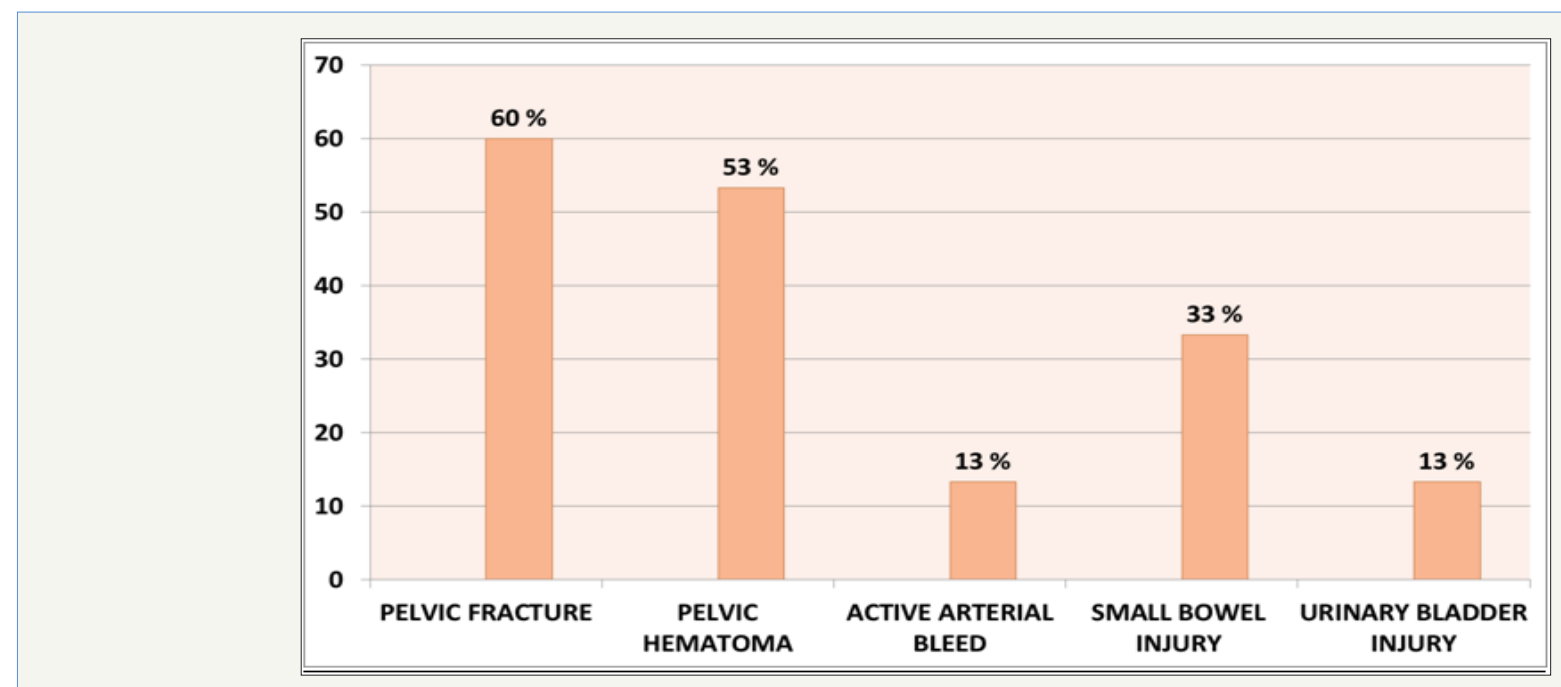

Figure 1: Other Injuries Associted with Extra-peritoneal Rectal Injury.

Nine patients $(60 \%)$ had pelvic fracture associated with rectal injury. Other injuries associated with rectal injury identified on CT abdomen and pelvis is shown in Figure I.

Diversion stoma was made in all of our patients. While diversion colostomy was the only surgical intervention in six patients, other patients were managed according to their injury patterns and decision of operating surgeon. Surgical treatments offered are as shown in Figure 2. 


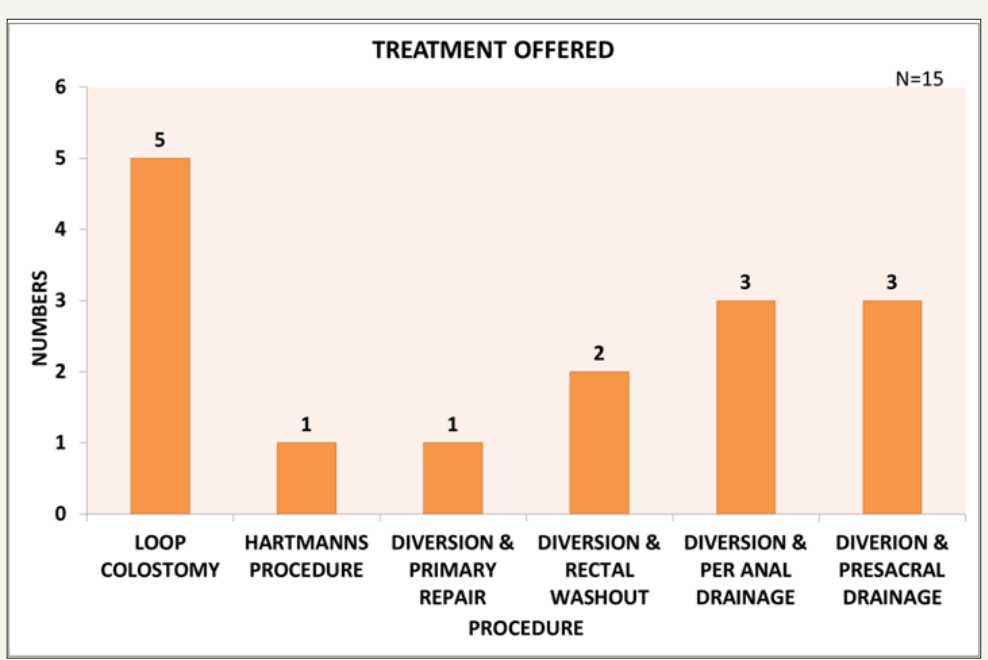

Figure 2: Surgical Treatment offered to patients suffering from Extra-peritoneal Rectal Injury.

Overall post-operative morbidity was $40 \%$. Two patients developed necrotizing fasciitis and required repeated debridements followed by graft placement and one patient developed intraabdominal abscess which was treated by radiological guided drain placement and antibiotics. Age greater than 40 years was found to be significantly associated with occurrence of post-operative complications ( $\mathrm{p}$-value $<0.01$ ).

\section{Discussion}

There is no defined management protocol for the treatment of civilian extra-peritoneal rectal injuries. Treatment involves a combination of techniques learnt from high-energy injuries during military conflicts which includes four main components:

i. Fecal diversion

ii. Pre sacral drainage

iii. Distal rectal washout; and

iv. Primary repair if possible.

Since the injuries in civilian trauma are low-energy, direct adaptation of these principles to civilian injuries is questionable.

The morbidity and mortality rates after rectal injuries are high. In a civilian rectal injury series these have been reported to be $6-42 \%$ and $0-10 \%$ respectively [8]. In a more recent study performed on 19 civilian patients with extra-peritoneal rectal injuries and 4 civilian patients with both intra and extra-peritoneal rectal injuries, Shatnawi and Bani-Hani reported the morbidity and mortality rates as $47.8 \%$ and $13 \%$, respectively [9]. This is comparable to our study where we had a morbidity rate of $40 \%$ and $0 \%$ mortality.

Missed injuries can lead to detrimental outcomes for example necrotizing fasciitis. In patients suspected to have rectal injury, a computed tomography (CT) scanning is recommended [10].

In the treatment of extraperitoneal rectal injuries, a diverting colostomy has been accepted as the standard therapy by many authors [11-13]. It has been reported that extraperitoneal rectal injuries can be safely treated with fecal diversion alone, particularly in low-velocity trauma $[14,15]$. Bostick et al. [11] reported that no septic complications were observed in any of the cases that underwent loop colostomies [11]. Demirbaş et al [16] verified the therapeutic approach consisting of a diverting colostomy (by performing a loop colostomy on all patients), distal rectal washout and pre sacral drainage in the treatment of ano-rectal gunshot injuries [16]. In our study out of fifteen patients except for one who required a Hartmann's procedure for technical reasons all underwent loop colostomy along with other options as mentioned earlier. Anterior lower one-third and posterior lower two-thirds rectal injuries are extra peritoneal and can be managed by primary repair on a case-by-case basis [17]. Some authors have suggested that primary repair without diversion is feasible in selected patients [18-20]. In a study involving 30 patients with extra peritoneal rectal injuries, Levine et al. [20] suggested that primary repair without fecal diversion could be considered in patients without major associated injuries when they were treated within 8 hours of injury and had rectal injury scores (RIS) $\leq 2$. However, the repair of extra peritoneal rectal perforations is not always technically feasible, and there is very little evidence to support the primary repair of these injuries [15]. Fecal diversion without primary repair is a safe procedure due to the anatomic considerations and technically difficult dissections. Those who advocate fecal diversion have suggested that the incidence of septic complications is less with diversion and have also shown that the incidence of stoma closure is associated with acceptable morbidity [21]. Primary repair is recommended in only one-half of the cases with extra peritoneal rectal injuries if it is possible to locate the wound [11]. The success rate of higher as compared to military series [10].

\section{Conclusion}

Drainage with fecal diversion is the most commonly employed management of extra-peritoneal rectal injuries. Delayed or inadequate drainage can lead to disastrous consequences including necrotizing fasciitis. 


\section{References}

1. Navsaria PH, Edu S, Nicol AJ (2011) Nonoperative management of pelvic gunshot wounds. Am J Surg 201(60): 784-788.

2. Loria F (1948) Historical aspects of penetrating wounds of the abdomen. Surg Gynecol Obstet 87(6): 521-549.

3. Wallace CMG (1916) A study of 1200 cases of gunshot wounds of the abdomen. British Journal of Surgery 4(16): 679-743.

4. Ogilvie W (1946) Abdominal wounds in the Western Desert. Bull US Army Med Dep 6(4): 435-445.

5. Lavenson GS, Cohen A (1971) Management of rectal injuries. Am J Surg 122(2): 226-230.

6. Shahzad N, Zafar H, Ur Rehman Z, Pardhan A (2017) Quality of Life after Damage Control Laparotomy for Trauma. Ann Surg Perioper Care 2(3): 1033.

7. Choi WJ (2011) Management of colorectal trauma. J Korean Soc Coloproctol 27(4): 166-172.

8. Burch J, Feliciano DV, Mattox KL (1989) Colostomy and drainage for civilian rectal injuries: is that all? Ann surg 209(5): 600-610.

9. Shatnawi NJ, Bani Hani KE (2006) Management of civilian extra peritoneal rectal injuries. Asian J Surg 29(1): 11-16.

10. Steele S R, Wolcott K E, Mullenix P S, Martin M J, Sebesta J A, et al. (2007) Colon and rectal injuries during Operation Iraqi Freedom: are there any changing trends in management or outcome? Dis Colon Rectum 50(6): 870-877.

11. Bostick PJ, Johnson DA, Heard JF, Islas JT, Sims EH, et al. (1993) Management of extraperitoneal rectal injuries. J Natl Med Assoc 85(6):460-463
12. Navsaria PH, Graham R, Nicol A (2001) A new approach to extra peritoneal rectal injuries: laparoscopy and diverting loop sigmoid colostomy. J Trauma 51(3): 532-535.

13. Navsaria PH, Shaw J, Zellweger R, Nicol A, Kahn D (2004) Diagnostic laparoscopy and diverting sigmoid loop colostomy in the management of civilian extraperitoneal rectal gunshot injuries. Br J Surg 91(4): 460 464

14. Velmahos GC, Gomez H, Falabella A, Demetriades D (2000) Operative management of civilian rectal gunshot wounds: simpler is better. World J Surg 24(1): 114-118.

15. Navsaria PH, Edu S, Nicol AJ (2007) Civilian extra peritoneal rectal gunshot wounds: surgical management made simpler. World J Surg 31(6): 1347-1353.

16. Yavuz K, Ereno C, Lu M, Akin ML Çelenk T, et al. (2004) Surgical treatment of ano-rectal gunshot injuries caused by low-velocity bullets. Turkish Journal of Trauma \& Emergency Surgery 10(1): 17-21.

17. Cleary RK, Pomerantz RA, Lampman RM (2006) Colon and rectal injuries. Dis Colon Rectum 49(8): 1203-1222.

18. Thomas DD, Levison M, Dykstra B, Bender J (1990) Management of rectal injuries. Dogma versus practice. The American Surgeon 56(8): 507-510.

19. Ivatury RR, Licata J, Gunduz Y, Rao P, Stahl W (1991) Management options in penetrating rectal injuries. Am surg 57(1): 50-55.

20. Levine J H, Longo W E, Pruitt C, Mazuski J E, Shapiro M J, Durham R M (1996) Management of selected rectal injuries by primary repair. Am J Surg 172(5): 575-579.

21. Berne JD, Velmahos GC, Chan LS, Asensio JA, Demetriades D (1998) The high morbidity of colostomy closure after trauma: further support for the primary repair of colon injuries. Surgery 123(2): 157-164.
Creative Commons Attribution 4.0

International License

For possible submissions Click Here

\section{Submit Article}

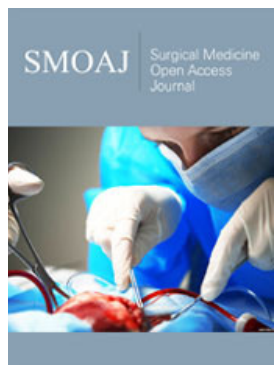

\section{Surgical Medicine Open Access Journal}

\section{Benefits of Publishing with us}

- High-level peer review and editorial services

- Freely accessible online immediately upon publication

- Authors retain the copyright to their work

- Licensing it under a Creative Commons license

- Visibility through different online platforms 